\title{
Extra Virgin Olive Oil Polyphenols Promote Cholesterol Efflux and Improve HDL Functionality
}

\author{
Hicham Berrougui, ${ }^{1,2}$ Souad Ikhlef, ${ }^{1}$ and Abdelouahed Khalil ${ }^{1}$ \\ ${ }^{1}$ Department of Medicine, Geriatrics Service, Faculty of Medicine and Biological Sciences, University of Sherbrooke, \\ $300112 e$ Avenue Nord, Sherbrooke, QC, Canada J1H 5N4 \\ ${ }^{2}$ Department of Biology, Polydisciplinary Faculty, University Sultan Moulay Slimane, BP 592, 23000 Beni Mellal, Morocco
}

Correspondence should be addressed to Hicham Berrougui; hicham.berrougui@usherbrooke.ca and Abdelouahed Khalil; a.khalil@usherbrooke.ca

Received 11 May 2015; Revised 27 June 2015; Accepted 2 July 2015

Academic Editor: Da-zhuo Shi

Copyright (C) 2015 Hicham Berrougui et al. This is an open access article distributed under the Creative Commons Attribution License, which permits unrestricted use, distribution, and reproduction in any medium, provided the original work is properly cited.

Results of the present work give evidence from the beneficial role of extra virgin olive of oil (EVOO) consumption towards oxidative stress and cardiovascular diseases. Polyphenols contained in EVOO are responsible for inhibiting lipoproteins oxidative damages and promoting reverse cholesterol transport process via ABCA1 pathway.

\section{Introduction}

Coronary heart disease (CHD) is the main cause of mortality in the Western world. The oxidation of low-density lipoproteins (LDL) is an early event in the development of atherosclerosis, the underlying cause of CHD [1]. Oxidized LDL are not recognized by the LDL-receptor Apo $(\mathrm{B} / \mathrm{E})$ but are taken up by macrophages in a nonregulated manner through the scavenger-receptor pathway, which leads to the formation of foam cells, the hallmark of arteriosclerotic lesions [1].

Macrophage-specific reverse cholesterol transport (RCT) is thought to be one of the most important HDL-mediated cardioprotective mechanisms. RCT is the process by which cholesterol in peripheral cells is effluxed onto circulating HDL and is transported back to the liver for excretion in bile and feces $[2,3]$. The promotion of RCT is considered a major antiatherogenic function of HDL $[4,5]$. The efflux of cholesterol from cells to HDL is the first and rate-limiting step of RCT [6]. Two major macrophages cholesterol efflux pathways have been described: SR-BI receptor-mediated cholesterol efflux and ABCA1/ABCG1-mediated cholesterol efflux. $A B C A 1$ promotes the efflux of phospholipids and cholesterol to lipid-poor apo-AI via a process that involves the direct binding of apo-AI to the ABCA1 transporter, whereas ABCG1 and SR-BI are key mediators of macrophage cholesterol efflux to mature HDL [7]. Evidence from a recent study indicates that the inflammatory process induces changes in HDL composition and metabolism that impair RCT [8]. Interestingly, we recently showed that RCT is also impaired with aging, especially by changes to the ABCA1-mediated cholesterol efflux pathway $[9,10]$.

Polyphenol-rich vegetable oils and monounsaturated fatty acids provide protection against an array of human diseases such as cancer, atherosclerosis, and CVD, including those involving the central nervous system. Olive oil, which is known for its healthful properties, which are often attributed to its high monounsaturated fatty acid content, including oleic acid (18:1 n-9), is a prominent member of the family of polyphenol- and monounsaturated fatty acid-rich oils. However, olive oil, unlike other vegetable oils, contains high amounts of several micronutrient constituents, including polyphenolic compounds $(100-1000 \mathrm{mg} / \mathrm{Kg}$ ) such as hydroxytyrosol, tyrosol, and oleuropein [11]. In vitro and in vivo human and animal studies have shown that EVOO reduces blood pressure [12], improves the lipid profile by increasing HDL-cholesterol and reducing LDL-cholesterol and triglyceride levels [13-15], reduces oxidative stress, and inhibits human lipoprotein oxidation, making LDL, for instance, less atherogenic $[16,17]$. Olive oil dietary supplementation decreases the levels of high inflammatory and endothelial 
dysfunction markers in the serum. Experimental and clinical studies have shown that olive oil downregulates vascular cell adhesion molecule-1 (VCAM-1), human soluble intercellular adhesion molecule-1 (sICAM-1), and E-selectin expression in the endothelium [18] and decreases plasma levels of sICAM1, soluble E-selectin, interleukin-6 (IL-6), and high-sensitive C-reactive protein (CRP) in high-risk patients [19].

The beneficial effects of polyphenols appear to be mediated via a plethora of biochemical pathways and signaling mechanisms that act either independently or synergistically. In the present study, we investigated the atheroprotective effect of the phenolic compounds in EVOO on cholesterol efflux and on oxidative stress damage in healthy subjects.

\section{Methods}

2.1. Subjects. Twenty-four healthy volunteers $(30.92 \pm 2.55$ years) with normal serum lipid profiles and blood pressure were recruited. They were all nonsmokers and were not taking any medication, including lipid-lowering treatments or oral antioxidants. None of the female subjects was undergoing estrogen replacement therapy for menopause. None of the participants showed clinical signs of inflammation, obesity, or diabetes. The physical and biochemical parameters of the participants are presented in Table 1. The Ethics Committee of the Sherbrooke Geriatric University Institute approved the study, and all subjects provided written informed consent before being enrolled.

2.2. Phytochemistry. The phenolic compounds were extracted from EVOO using the method of Pirisi et al. [20]. Briefly, EVOO was mixed with $n$-hexane and methanol/water and was stirred in a vortex apparatus overnight at $4^{\circ} \mathrm{C}$. The mixture was then centrifuged, and the hydroalcoholic solution was washed with $n$-hexane and then lyophilized overnight.

2.3. Lipoprotein Isolation. Fasting human plasma was collected in heparin tubes, and the HDL was immediately isolated using the method of Sattler et al. [21]. The isolated lipoproteins were dialyzed overnight at $4^{\circ} \mathrm{C}$ against $10^{-2} \mathrm{M}$ sodium phosphate buffer ( $\mathrm{pH} 7.0$ ). The protein concentrations were measured using commercial assay kits (Bio-Rad, Canada) using the manufacture's protocol.

\subsection{Lipoprotein Enrichment with EVOO and EVOO-PC.} Human plasma was incubated overnight with slight agitation at $4^{\circ} \mathrm{C}$ in the presence of EVOO $(0.2 \mathrm{mg} / \mathrm{mL}$ of plasma $)$ or EVOO-PC $(1.76 \mathrm{mg} / \mathrm{mL}$ of plasma). The LDL and HDL were then isolated as described above.

2.5. Copper-Mediated Lipoprotein Oxidation. The lipoproteins were peroxidized as previously described using transition metal ions as oxidizing agents [22]. Briefly, control, EVOO, and EVOO-PC-enriched lipoproteins [(LDL $100 \mu \mathrm{g} / \mathrm{mL}$ ) or (HDL $200 \mu \mathrm{g} / \mathrm{mL}$ )] were suspended in $10 \mathrm{mM}$ sodium phosphate buffer $(\mathrm{pH} 7)$ and were incubated for 0 to $4 \mathrm{~h}$ at $37^{\circ} \mathrm{C}$ in the presence of $10 \mu \mathrm{M}$ cupric sulfate. The oxidation reaction was stopped by adding EDTA. Lipid
TABLE 1: Clinical and biochemical parameters of participants.

\begin{tabular}{lc}
\hline & Mean \pm esm \\
\hline$n=24(\mathrm{w} / \mathrm{m})$ & $14 / 10$ \\
Age $(\mathrm{mean} \pm \mathrm{SD}$ years) & $30.92 \pm 2.55$ \\
Body mass index $\left(\mathrm{kg} / \mathrm{m}^{2}\right)$ & $23.7 \pm 1.65$ \\
System blood pressure $(\mathrm{mmHg})$ & $127 \pm 4.65$ \\
Dias. blood pressure $(\mathrm{mmHg})$ & $78.23 \pm 2.09$ \\
Total cholesterol (mmol/L) & $5.06 \pm 0.2$ \\
Triglycerides $(\mathrm{mmol} / \mathrm{L})$ & $1.32 \pm 0.15$ \\
HDL-c (mmol/L) & $1.42 \pm 0.09$ \\
LDL-c (mmol/L) & $3.05 \pm 0.15$ \\
Apo Al (g/L) & $1.56 \pm 0.05$ \\
Apo B (g/L) & $0.90 \pm 0.04$ \\
Apo B/Apo A1 & $0.8 \pm 0.04$ \\
TC/HDL-c & $3.81 \pm 0.23$ \\
LDL-c/HDL-c & $2.5 \pm 0.2$ \\
TG/HDL-c & $1.08 \pm 0.17$ \\
Glucose (mmol/L) & $4.43 \pm 0.10$ \\
Insulin (pmol/L) & $38.32 \pm 5.26$ \\
CRP (mg/L) & $3.16 \pm 0.13$ \\
\hline TC(total
\end{tabular}

TC (total cholesterol); HDL-C (HDL-cholesterol); LDL-C (LDL-cholesterol); CRP (C-reactive protein).

peroxide formation was assessed by monitoring conjugated diene formation at $234 \mathrm{~nm}$.

2.6. Cell Cultures. Human THP-1 monocytes and J774 macrophages were cultured in RPMI 1640 and DMEM medium, respectively. The media were supplemented with $10 \%$ heatinactivated FBS, $50 \mathrm{mM}$ 2- $\beta$-mercaptoethanol (only for THP1), $2 \mathrm{mM}$ L-glutamine, $5 \mathrm{mg} / \mathrm{mL}$ of glucose, and $100 \mathrm{U} / \mathrm{mL}$ of penicillin. The differentiation of the THP-1 monocytes into macrophages was induced by culturing the monocytes in the presence of $100 \mu \mathrm{M}$ PMA for $96 \mathrm{~h}$.

2.7. Cholesterol Efflux Measurements. THP-1-derived macrophages and $\mathrm{J774}$ macrophages were incubated in fresh growth medium containing $0.2 \mu \mathrm{Ci} / \mathrm{mL}\left[{ }^{3} \mathrm{H}\right]$-cholesterol for $48 \mathrm{~h}$ or $1 \mu \mathrm{Ci} / \mathrm{mL}\left[{ }^{3} \mathrm{H}\right]$-cholesterol for $24 \mathrm{~h}$, respectively. The loaded cells were washed, equilibrated in serum-free medium containing $1 \%$ BSA for $12 \mathrm{~h}$, washed again, and subjected to various treatments. The THP-1-derived macrophages were incubated for $24 \mathrm{~h}$ with (1) HDL-free medium, (2) HDL (50 $\mu \mathrm{g} / \mathrm{mL})$, (3) EVOO-enriched HDL (OO-HDL), or (4) EVOO-PC-enriched HDL (PC-HDL).

$\left[{ }^{3} \mathrm{H}\right]$-Cholesterol loaded THP-1-derived macrophages were subjected to oxidative stress by incubating them with $0.2 \mathrm{mM}$ iron/ascorbate (Fe/Asc) in the absence or presence of EVOO-PC $(320 \mu \mathrm{g} / \mathrm{mL})$ for $6 \mathrm{~h}$. They were then incubated with HDL for $24 \mathrm{~h}$ to assess cholesterol efflux under various conditions.

The effect of EVOO-PC on ABCA1-mediated cholesterol efflux was assessed using J774 macrophages. $\left[{ }^{3} \mathrm{H}\right]$-Cholesterol-loaded J774 macrophages were incubated for $12 \mathrm{~h}$ with 0 to $320 \mu \mathrm{g} / \mathrm{mL}$ of EVOO-PC to generate ABCA1-enriched cells 
or with $300 \mu \mathrm{M} 8$-Br-cAMP (positive control) to stimulate ABCA1 gene transcription and surface protein expression. The J7774 macrophages were then incubated with $25 \mu \mathrm{g} / \mathrm{mL}$ of apo-AI for $4 \mathrm{~h}$.

To better understand the mechanism of EVOO-PCmediated cholesterol efflux, we studied the effect of the two major phenolic compounds in EVOO (tyrosol and hydroxytyrosol) on ABCA1-mediated cholesterol efflux. $\left[{ }^{3} \mathrm{H}\right]$-Cholesterol-loaded J774 macrophages were incubated for $12 \mathrm{~h}$ with 0 to $25 \mu \mathrm{M}$ tyrosol or hydroxytyrosol to generate ABCA1enriched cells and were then incubated with $25 \mu \mathrm{g} / \mathrm{mL}$ of apoAI for 4 h. 8-Br-cAMP was used as a positive control.

Cholesterol efflux was determined by liquid scintillation counting, and the percent of radiolabeled cholesterol released (percent cholesterol efflux) was calculated using the following formula: (cpm in the medium/[cpm in the cells + medium $]) \times$ 100.

2.8. Western Blot Analyses. ABCA1 protein expression in J774 macrophages was studied by incubating them for $12 \mathrm{~h}$ with 0 to $320 \mathrm{mg} / \mathrm{mL}$ of EVOO-PC or 5 or $10 \mu \mathrm{M}$ hydroxytyrosol or tyrosol. The proteins $(20 \mu \mathrm{g})$ were separated by electrophoresis on $10 \%$ acrylamide gels and were transferred to polyvinylidene difluoride (PVDF) membranes. The membranes were blocked with 5\% milk in PBS/Tween 20 and were incubated with primary antibodies (anti-ABCA1) and then with specific IgG-HRP-conjugated secondary antibodies. $\beta$ actin was used as a control. The protein bands were detected using an enhanced chemiluminescence reagent (ECL) [10].

2.9. Statistical Analysis. Values are expressed as means \pm SEM. A one-way analysis of variance (ANOVA) was used for multiple comparisons. A linear regression analysis was used to assess the association between two continuous variables. All statistical analyses were performed using GraphPad Prism-5 software.

\section{Results}

3.1. Effect of Extra Virgin Olive Oil and EVOO Phenolic Compound Extracts on Lipoprotein Oxidation. The concentration of total phenolic compounds $(41.9 \mathrm{mM}$; gallic acid equivalent) was estimated using the Folin-Ciocalteu method.

The peroxidation by $\mathrm{CuSO}_{4}$ of the polyunsaturated fatty acids (PUFA) in HDL and LDL was assessed by the formation of conjugated dienes. The peroxidation kinetics showed that the lag phase of LDL was longer than that of HDL. The lag phase was followed by the propagation and termination phases.

Our results showed that plasma LDL and HDL that had been pretreated with EVOO or EVOO-PC were less oxidizible and were much more resistant to lipid peroxidation than untreated (control) plasma LDL and HDL as shown by the significant increase in the lag phase and the decrease in conjugated diene formation in EVOO-PC- and EVOO-treated lipoproteins (Figures 1(b) and 1(e)). The enrichement of lipoproteins with EVOO-PC or EVOO increased the lag phase 1.42- $(p<0.05)$ and 2.39 -fold $(p<0.01)$ for HDL and
1.51- and 1.50-fold ( $p<0.05$ ) for LDL, respectively, compared to the control (Figures 1(b) and 1(e)). On the other hand, the enrichment of HDL and LDL with EVOO or EVOO$\mathrm{PC}$ reduced conjugated diene formation $\left(\mathrm{OD}_{\max }\right) 4.53-(p<$ $0.05)$ and 7.71-fold $(p<0.01)$ for HDL and 1.75- $(p<0.001)$ and 14.58-fold ( $p<0.0001)$ for LDL, respectively, compared to the control (Figures 1(c) and 1(f)).

3.2. Effect of Phenolic Compounds on Reverse Cholesterol Transport. To determine the effect of phenolic compounds on RCT, cholesterol efflux was measured. Incubating ${ }^{3} \mathrm{H}$ cholesterol-loaded THP-1-derived macrophages for $24 \mathrm{~h}$ with EVOO or EVOO-PC enhanced cholesterol efflux by $41.5 \%$ and $39.93 \%(p<0.05)$, respectively, compared to the control (Figure 2(a)).

Oxidative damage to macrophages impairs cholesterol efflux, as shown by the decrease in ABCA1 protein expression induced by $\mathrm{Fe} / \mathrm{Asc}$ [23]. We thus investigated the effect of EVOO-PC on the capacity of HDL to mediate cholesterol efflux in THP-1-derived macrophages under oxidative stress induced by Fe/Asc. HDL-mediated cholesterol efflux was significantly impaired under oxidative stress conditions whereas the effect was much lower when the macrophages were pretreated with $320 \mu \mathrm{g} / \mathrm{mL}$ of EVOO-PC $(p<0.001)$ (Figure 2(b)).

To better understand the mechanism by which EVOO-PC enhances HDL-mediated cholesterol efflux, we investigated the effect of EVOO-PC on ABCA1-dependent cholesterol efflux from J774 macrophages. ${ }^{3} \mathrm{H}$-Cholesterol-loaded J774 macrophages were incubated with apoA-1 in the absence of cAMP for $4 \mathrm{~h}$ (time range for measuring cholesterol efflux via the ABCA1 pathway). We observed little cholesterol efflux in the absence of cAMP. However, when the macrophages were preincubated overnight with 0 to $320 \mu \mathrm{g} / \mathrm{mL}$ of EVOO-PC or with a CAMP-analogue to induce ABCA1 protein expression and then with $25 \mu \mathrm{g} / \mathrm{mL}$ of apoA-1 for $4 \mathrm{~h}$, we observed a significant EVOO-PC concentration-dependent increase in cholesterol efflux $\left(r^{2}=0.95, p<0.01\right)$ (Figure 3(a)).

To investigate the mechanism by which EVOO-PC induces the increase in cholesterol efflux from J774 macrophages to apoA-1, we performed Western blot analyses to measure ABCA1 protein expression on J774 macrophages incubated with EVOO-PC. We observed an EVOO-PC concentration-dependent increase in ABCA1 protein expression in J774 macrophages incubated with EVOO-PC (Figure 3(b)).

In light of these results, we then investigated the effect of two major phenolic compounds in EVOO-PC (purified tyrosol and hydroxytyrosol) on cholesterol efflux from and ABCA1 protein expression in J774 macrophages. Our results showed that tyrosol and hydroxytyrosol increase in concentration-dependent manner the ABCA1-dependent cholesterol efflux (Figures 4(a) and 4(b), resp.).

\section{Discussion}

Olive oil is the main source of fat in the Mediterranean diet. A large body of knowledge has provided evidence of the benefits of the Mediterranean diet and olive oil consumption on 


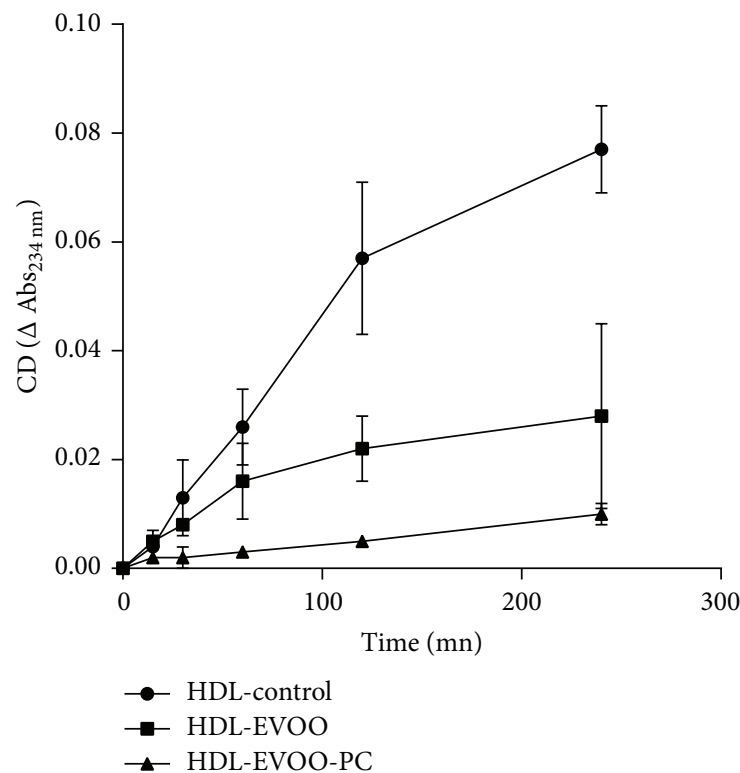

(a)

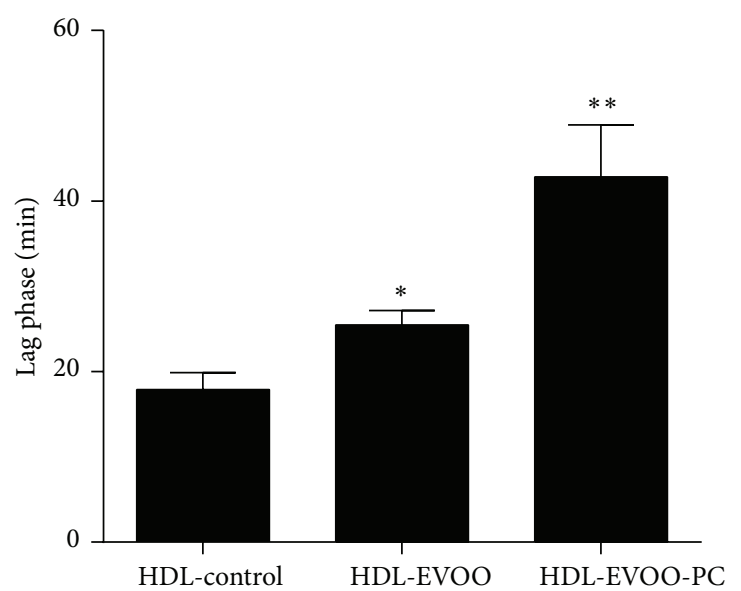

(b)

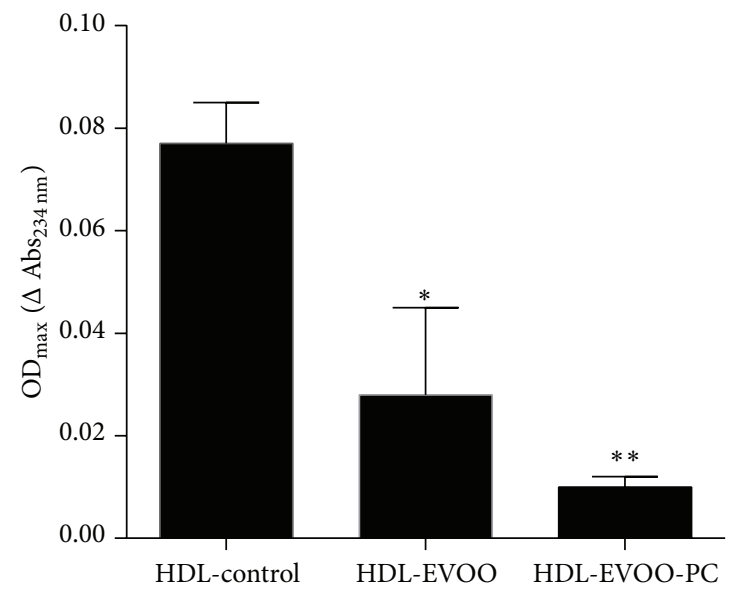

(c)

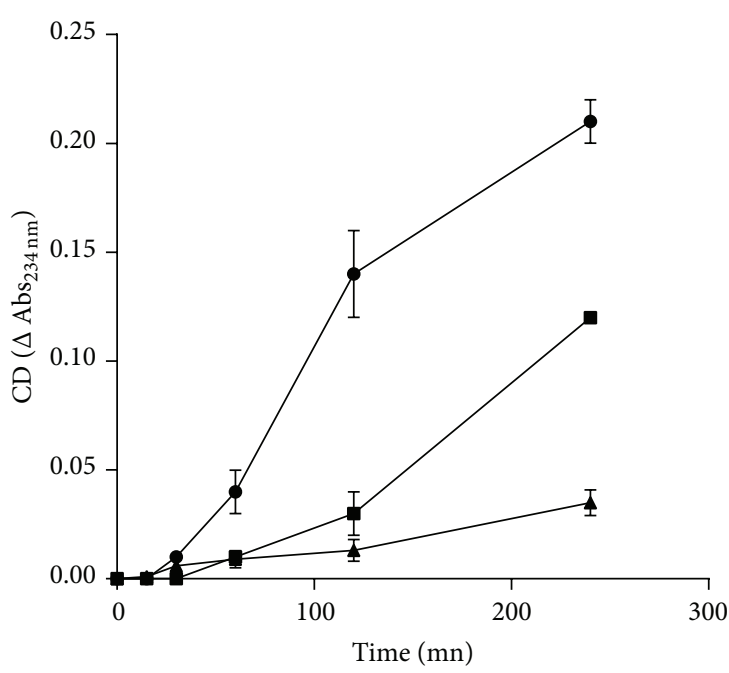

$\rightarrow$ LDL-control

$\rightarrow$ LDL-EVOO LDL-EVOO-PC

(d)

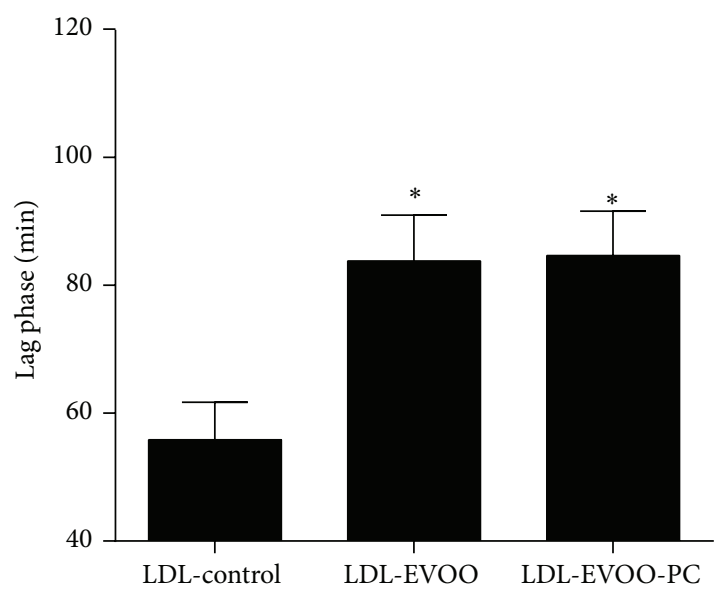

(e)

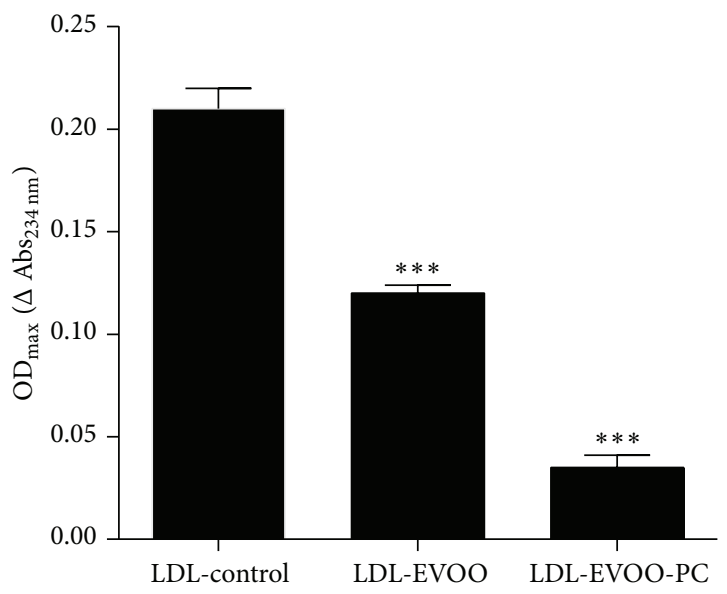

(f)

FIGURE 1: EVOO-PC enrichment decreases the oxidizability of lipoproteins. Plasma was incubated with EVOO or EVOO-PC prior to isolating HDL and LDL. HDL and LDL pretreated with EVOO or EVOO-PC as well as untreated controls were oxidized by incubation with copper ions for $4 \mathrm{~h}$. The resistance to lipid peroxidation and the oxidizability of HDL and LDL were monitored by determining the lag phase (a, b, $d$, e) and by measuring conjugated diene formation $\left(\mathrm{OD}_{\max }\right)$, respectively $(c, f)$. Results are expressed as the means \pm SEM of three independent experiments. ${ }^{*} p<0.05,{ }^{* *} p<0.01$, and ${ }^{* * *} p<0.001$. 


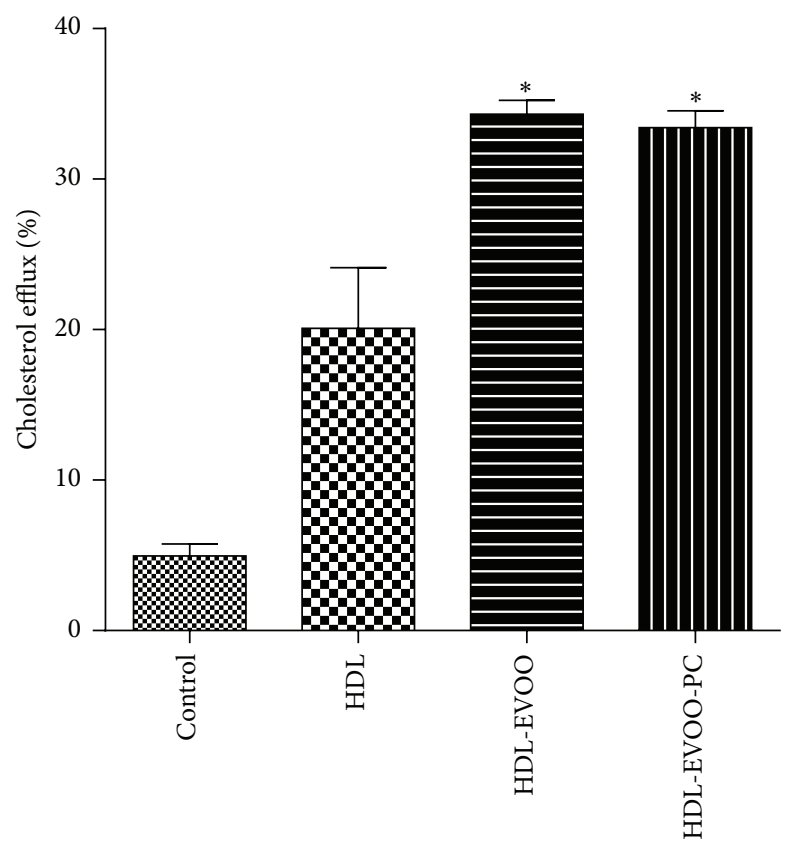

(a)

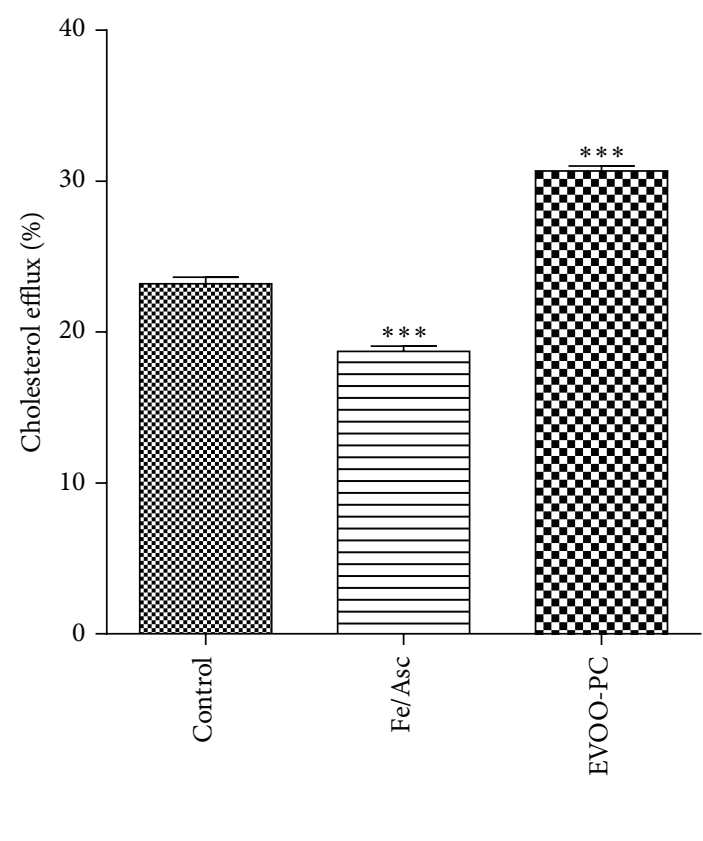

(b)

FIGURE 2: EVOO-PC protects macrophages against oxidation and promotes HDL-mediated cholesterol efflux. (a) THP-1-derived macrophages were loaded with $\left[{ }^{3} \mathrm{H}\right]$-cholesterol $(2 \mu \mathrm{Ci} / \mathrm{mL})$ for $24 \mathrm{~h}$. The cells were then washed, equilibrated, and incubated for a further $24 \mathrm{~h}$ with $50 \mu \mathrm{g} / \mathrm{mL}$ of HDL-free medium, HDL, EVOO-enriched HDL (EVOO-HDL), or EVOO-PC-enriched HDL (EVOO-PC-HDL). (b) The macrophages were stressed with $0.2 \mathrm{mM} \mathrm{Fe} /$ Asc, and cholesterol efflux was assessed using $50 \mu \mathrm{g} / \mathrm{mL}$ of HDL. Results are expressed as the means \pm SEM of at least three independent experiments. ${ }^{*} p<0.05,{ }^{* *} p<0.001$, and ${ }^{* * *} p<0.001$.

the prevention of atherosclerosis and CHD [24-27]. Several studies have reported that the antiatherogenic effect of olive oil is related to the antioxidant and anti-inflammatory effects exerted by various components, especially monounsaturated fatty acids (MUFA) and polyphenols [11, 17, 28-30]. Phenolic compounds, especially hydroxytyrosol and oleuropein, dosedependently inhibit LDL and HDL oxidation in vitro and in vivo, repress superoxide-driven reactions, and break the chain-like propagation of lipid peroxides [31-34]. Interestingly, a study by Covas et al. [17] showed that consuming EVOO increases the postprandial concentration of phenolic compounds in the plasma and in LDL and HDL, which may explain the protective effect of phenolic compounds.

Plasma HDL-cholesterol levels are markedly and inversely correlated to the risk of atherosclerotic cardiovascular diseases [35]. It has been suggested that HDL facilitates cholesterol efflux from peripheral tissues and transports it back to the liver in a process called RCT [36]. ABCA1 facilitates cholesterol efflux from cells to lipid-poor apo-AI but not to HDL $[7,37]$, whereas another ABC transporter, ABCG1, as well as the SR-BI receptor, is involved in cholesterol efflux from macrophages to HDL $[38,39]$. Some studies have suggested that food nutrients and diet may play pivotal roles in the regulation of RCT [25, 40-42]. We have previously shown that EVOO consumption improves the RCT process by enhancing the capacity of HDL to mediate cholesterol efflux and of human monocyte-derived macrophages (HMDM) to excrete free cholesterol [43]. In the present study, we investigated how the consumption of EVOO may promote cholesterol efflux. We focused on the effect of EVOO-PC, especially essential phenols such as tyrosol and hydroxytyrosol.

Our results showed that enriching LDL and HDL with EVOO-PC results in an increase in the resistance of LDL and HDL to lipid peroxidation. This effect may be due to the antioxidant effect of the phenolic compounds, which may scavenge reactive oxygen species and thus inhibit lipoprotein oxidation $[16,44,45]$. Incubating plasma with EVOOPC increased the binding of polyphenols to LDL and HDL lipoproteins, as previously reported by Covas et al. [17, 46] and Lamucla-Raventós et al. [47]. Moreover, in a recent study, Hernáez et al. [48] showed that olive oil polyphenols increase the size of HDL particles, enhance the stability of HDL by generating a triglyceride-poor core, and enhance the antioxidant status of HDL by increasing the olive oil polyphenol metabolite content of the lipoprotein. Olive oil polyphenols are highly bioavailable, which provides further support for their putative health-promoting effects (reviewed in $[49,50]$ ). However, very few studies have been conducted on the effect of phenolic compounds on RCT. Our results showed that EVOO-PC-enriched HDL promotes RCT by enhancing cholesterol efflux from THP-1-derived macrophages. This effect may be related to an improvement in the physicochemical properties of HDL by increasing their phenol content, which protects HDL from oxidation, and by increasing the fluidity of the phospholipidic layer. Indeed, we previously 

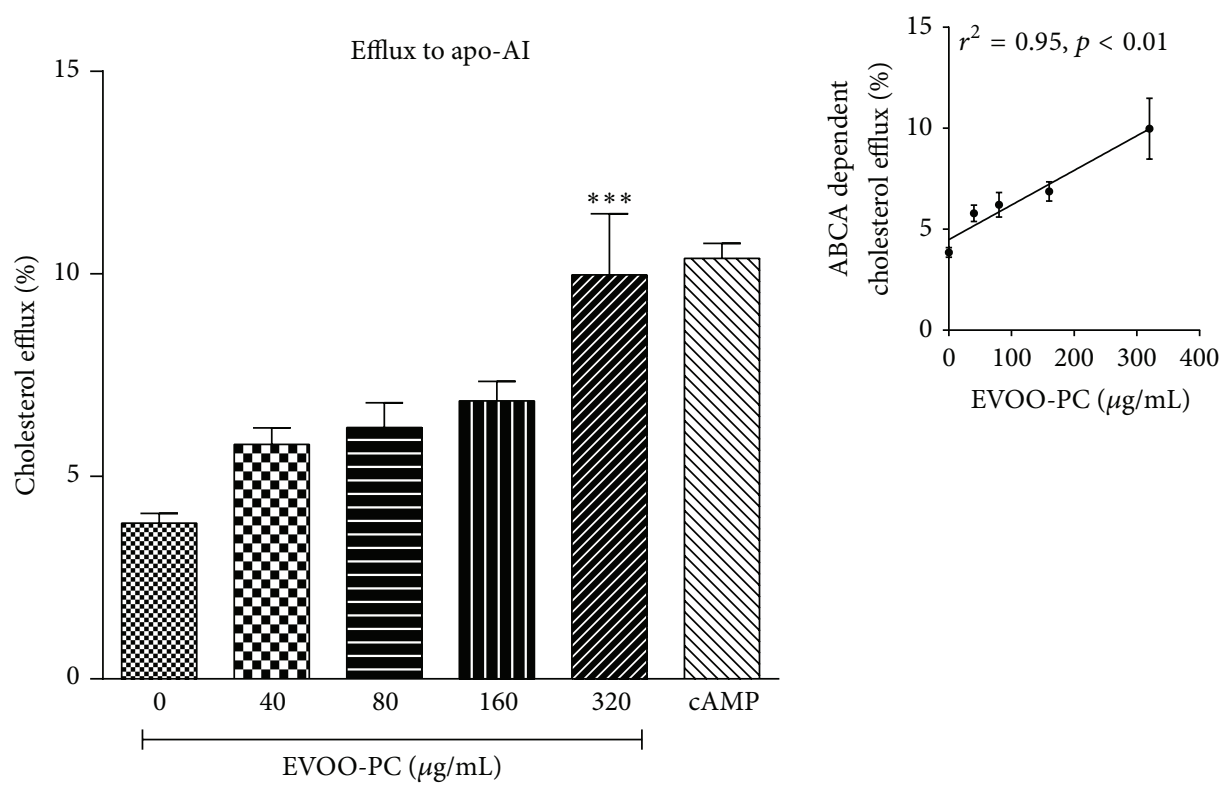

(a)

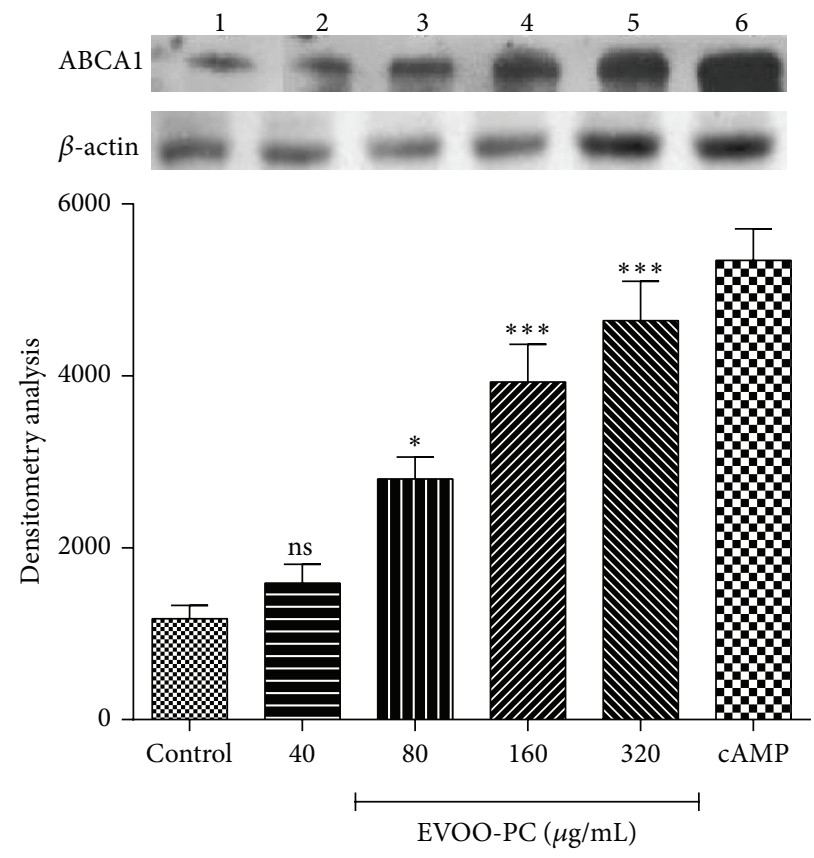

(b)

FIGURE 3: EVOO-PC increases ABCA1 protein expression and enhances apoA-I-mediated cholesterol efflux. (a) [ $\left.{ }^{3} \mathrm{H}\right]$-Cholesterol-loaded J774 macrophages were incubated for $12 \mathrm{~h}$ with various concentrations of EVOO-PC (0 to $320 \mu \mathrm{g} / \mathrm{mL}$ ) or with cAMP (positive control) to generate ABCA1-enriched cells, which were incubated with $25 \mu \mathrm{g} / \mathrm{mL}$ of apo-AI for $4 \mathrm{~h}$. The small upper panel shows the positive correlation between EVOO-PC concentrations and ABCA1-dependent cholesterol efflux. (b) ABCA1 protein expression after incubating J774 macrophages with increasing concentrations of EVOO-PC as determined by densitometric analyses of protein bands on PVDF membranes. Results are expressed as the means \pm SEM of at least three independent experiments. ${ }^{*} p<0.05,{ }^{* *} p<0.001$, and ${ }^{* * *} p<0.001$.

showed that polyphenol compounds from argan oil (a polyphenol-rich vegetable oil) also enhance HDL-mediated cholesterol efflux by improving HDL fluidity and increasing HDL binding to cell membranes [16]. In the present study, we also investigated the effect of EVOO-PC on cholesterol efflux from THP-1-derived macrophages stressed by Fe/Asc, which induces lipid peroxidation [51] and reduces cholesterol efflux. Pretreating macrophages with EVOO-PC before incubating them with $\mathrm{Fe} / \mathrm{Asc}$ significantly restored cholesterol efflux from macrophages to HDL, likely by suppressing the effect 


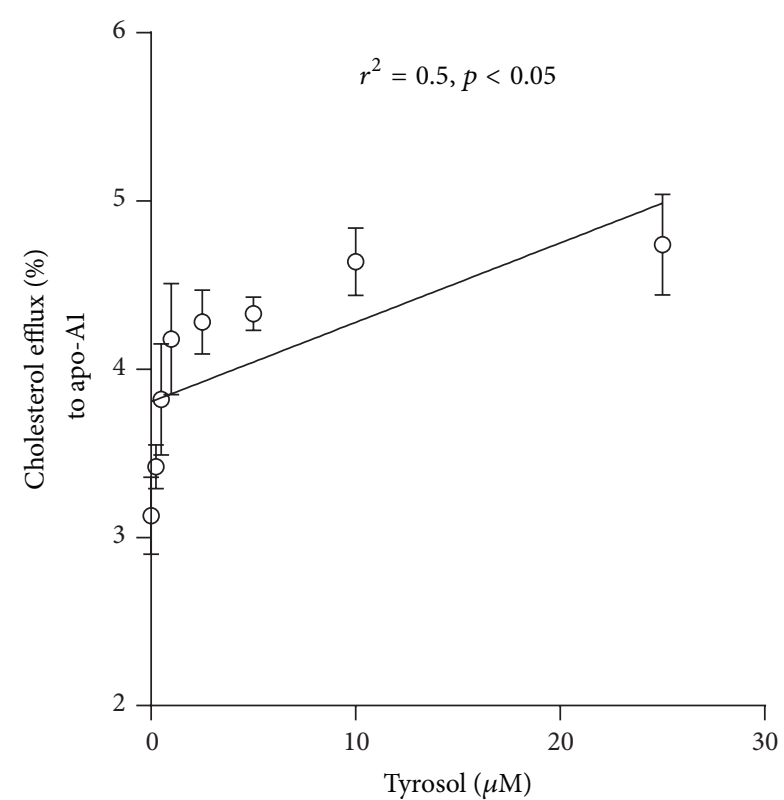

(a)

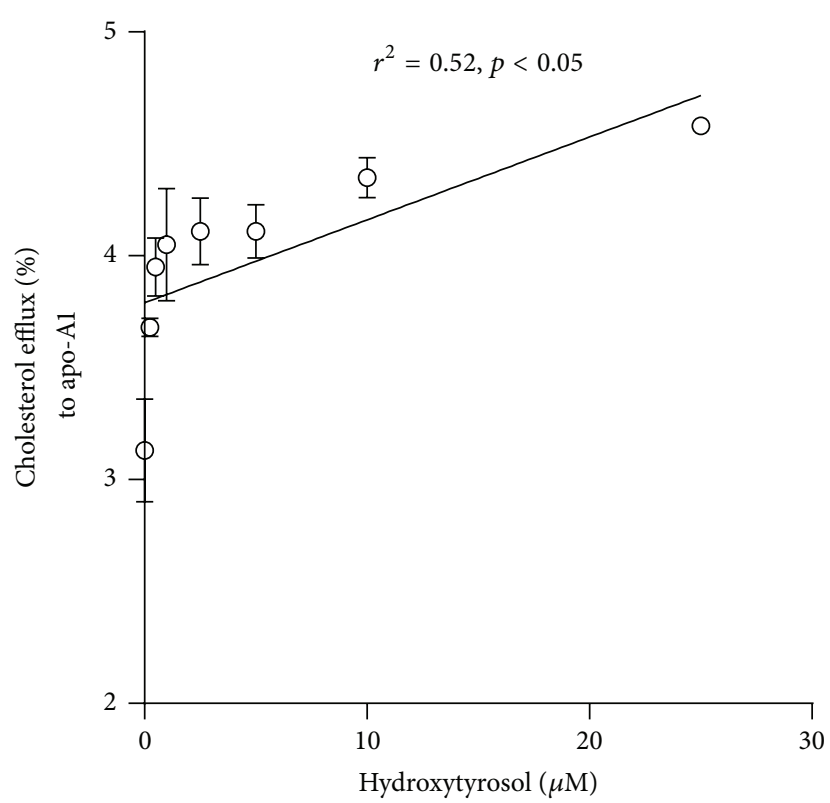

(b)

FIGURE 4: Tyrosol and hydroxytyrosol increase ABCA1 protein expression and enhance apoA-I-mediated cholesterol efflux. $\left[{ }^{3} \mathrm{H}\right]$-Cholesterolloaded J774 macrophages were incubated for $12 \mathrm{~h}$ with different concentrations ( 0 to $25 \mu \mathrm{M}$ ) of tyrosol (a) or hydroxytyrosol (b) to generate ABCA1-enriched cells, which were then incubated with $25 \mu \mathrm{g} / \mathrm{mL}$ of apo-AI for $4 \mathrm{~h}$. Results are expressed as the means \pm SEM of at least three independent experiments.

of $\mathrm{Fe} / \mathrm{Asc}$ on the cell surface receptors involved in this process. This effect has also been reported with vitamin $\mathrm{E}$ and butylhydroxytoluene (BHT), two other antioxidants [23].

However, little is known about the molecular mechanism by which phenolic compounds promote cholesterol efflux. To better understand the mechanism by which EVOO-PC enhances HDL-mediated cholesterol efflux, we investigated the effect of EVOO-PC on cell signaling pathways. Our results clearly showed that EVOO-PC, including tyrosol and hydroxytyrosol, stimulates ABCA1 protein expression in J774 macrophages, which may explain how these phenols promote cholesterol efflux to apoA-1. Uto-Kondo et al. [40] reported that coffee consumption by healthy humans enhances HDLmediated cholesterol efflux by increasing ABCG1 and SRBI but not ABCA1 expression and that this may be due to the phenolic acids in the coffee. This appears to be unlikely given that phenolic acids activate liver X receptor- $\alpha(\mathrm{LXR} \alpha)$ expression, which in turn transactivates both ABCA1 and ABCG1. However, other studies, including ours, have shown that resveratrol stimulates LXR $\alpha, \mathrm{ABCA} 1$, and ABCG1 [52, 53]. It thus appears that different phenolic compounds may stimulate cholesterol efflux via different mechanisms.

In conclusion, our results showed that EVOO-PC enhances the antiatherogenic properties of HDL by reducing oxidative modifications to HDL and by maintaining the physicochemical properties of HDL, which in turn improve the functionality of HDL, especially the capacity to promote cholesterol efflux. EVOO-PC also protected cells from oxidative damage and stimulated ABCA1 protein expression, a key factor in cholesterol efflux and HDL genesis. Our results are in agreement with our previous findings showing that the consumption of olive oil polyphenols helps to reduce cardiovascular risk.

\section{Conflict of Interests}

The authors declare that they have no conflict of interests regarding the publication of this paper.

\section{Acknowledgment}

The present study was supported by grants from the Canadian Institutes of Health Research (MOP-89912 and IAO-134212).

\section{References}

[1] M. A. Ghaffari and T. Ghiasvand, "Kinetic study of low density lipoprotein oxidation by copper," Indian Journal of Clinical Biochemistry, vol. 25, no. 1, pp. 29-36, 2010.

[2] E. Favari, F. Zimetti, A. E. Bortnick et al., "Impaired ATP-binding cassette transporter A1-mediated sterol efflux from oxidized LDL-loaded macrophages," FEBS Letters, vol. 579, no. 29, pp. 6537-6542, 2005.

[3] X. Mei and D. Atkinson, "Lipid-free apolipoprotein A-I structure: insights into HDL formation and atherosclerosis development," Archives of Medical Research, 2015.

[4] A. Rohatgi, "High-density lipoprotein function measurement in human studies: focus on cholesterol efflux capacity," Progress in Cardiovascular Diseases, vol. 58, no. 1, pp. 32-40, 2015.

[5] S. W. Sakr, D. L. Williams, G. W. Stoudt, M. C. Phillips, and G. H. Rothblat, "Induction of cellular cholesterol efflux to lipid-free 
apolipoprotein A-I by cAMP," Biochimica et Biophysica Acta, vol. 1438, no. 1, pp. 85-98, 1999.

[6] J. C. Escolà-Gil, G. Llaverias, J. Julve, M. Jauhiainen, J. MéndezGonzález, and F. Blanco-Vaca, "The cholesterol content of western diets plays a major role in the paradoxical increase in highdensity lipoprotein cholesterol and upregulates the macrophage reverse cholesterol transport pathway," Arteriosclerosis, Thrombosis, and Vascular Biology, vol. 31, no. 11, pp. 2493-2499, 2011.

[7] N. Wang, D. L. Silver, C. Thiele, and A. R. Tall, "ATP-binding cassette transporter $\mathrm{Al}$ (ABCA1) functions as a cholesterol efflux regulatory protein," The Journal of Biological Chemistry, vol. 276, no. 26, pp. 23742-23747, 2001.

[8] F. C. McGillicuddy, M. L. de la Moya, C. C. Hinkle et al., "Inflammation impairs reverse cholesterol transport in vivo," Circulation, vol. 119, no. 8, pp. 1135-1145, 2009.

[9] H. Berrougui and A. Khalil, "Age-associated decrease of highdensity lipoprotein-mediated reverse cholesterol transport activity," Rejuvenation Research, vol. 12, no. 2, pp. 117-126, 2009.

[10] H. Berrougui, M. Isabelle, M. Cloutier, G. Grenier, and A. Khalil, "Age-related impairment of HDL-mediated cholesterol efflux," Journal of Lipid Research, vol. 48, no. 2, pp. 328-336, 2007.

[11] C. R. Sirtori, E. Gatti, E. Tremoli et al., "Olive oil, corn oil, and n-3 fatty acids differently affect lipids, lipoproteins, platelets, and Superoxide formation in type II hypercholesterolemia," American Journal of Clinical Nutrition, vol. 56, no. 1, pp. 113-122, 1992.

[12] J. S. Perona, J. Cañizares, E. Montero, J. M. Sánchez-Domínguez, A. Catalá, and V. Ruiz-Gutiérrez, "Virgin olive oil reduces blood pressure in hypertensive elderly subjects," Clinical Nutrition, vol. 23, no. 5, pp. 1113-1121, 2004.

[13] M.-I. Covas, K. Nyyssönen, H. E. Poulsen et al., "The effect of polyphenols in olive oil on heart disease risk factors: a randomized trial," Annals of Internal Medicine, vol. 145, no. 5, pp. 333341, 2006.

[14] A. Blanco-Molina, G. Castro, D. Martín-Escalante et al., "Effects of different dietary cholesterol concentrations on lipoprotein plasma concentrations and on cholesterol efflux from Fu5AH cells," The American Journal of Clinical Nutrition, vol. 68, no. 5, pp. 1028-1033, 1998.

[15] M. A. Carluccio, M. Massaro, E. Scoditti, and R. De Caterina, "Vasculoprotective potential of olive oil components," Molecular Nutrition and Food Research, vol. 51, no. 10, pp. 1225-1234, 2007.

[16] H. Berrougui, M. Cloutier, M. Isabelle, and A. Khalil, "Phenolicextract from argan oil (Argania spinosa L.) inhibits human lowdensity lipoprotein (LDL) oxidation and enhances cholesterol efflux from human THP-1 macrophages," Atherosclerosis, vol. 184, no. 2, pp. 389-396, 2006.

[17] M.-I. Covas, K. De La Torre, M. Farré-Albaladejo et al., "Postprandial LDL phenolic content and LDL oxidation are modulated by olive oil phenolic compounds in humans," Free Radical Biology \& Medicine, vol. 40, no. 4, pp. 608-616, 2006.

[18] M. Dell'Agli, R. Fagnani, N. Mitro et al., "Minor components of olive oil modulate proatherogenic adhesion molecules involved in endothelial activation," Journal of Agricultural and Food Chemistry, vol. 54, no. 9, pp. 3259-3264, 2006.

[19] B. Cortés, I. Núñez, M. Cofán et al., "Acute effects of highfat meals enriched with walnuts or olive oil on postprandial endothelial function," Journal of the American College of Cardiology, vol. 48, no. 8, pp. 1666-1671, 2006.
[20] F. M. Pirisi, P. Cabras, C. F. Cao, M. Migliorini, and M. Muggelli, "Phenolic compounds in virgin olive oil. 2. Reappraisal of the extraction, HPLC separation, and quantification procedures," Journal of Agricultural and Food Chemistry, vol. 48, no. 4, pp. 1191-1196, 2000.

[21] W. Sattler, D. Mohr, and R. Stocker, "Rapid isolation of lipoproteins and assessment of their peroxidation by highperformance liquid chromatography postcolumn chemiluminescence," Methods in Enzymology, vol. 233, pp. 469-489, 1994.

[22] A. Khalil, J.-P. Fortin, J.-G. LeHoux, and T. Fülöp, "Agerelated decrease of dehydroepiandrosterone concentrations in low density lipoproteins and its role in the susceptibility of low density lipoproteins to lipid peroxidation," Journal of Lipid Research, vol. 41, no. 10, pp. 1552-1561, 2000.

[23] V. Marcil, E. Delvin, A. T. Sané, A. Tremblay, and E. Levy, "Oxidative stress influences cholesterol efflux in THP-1 macrophages: role of ATP-binding cassette A1 and nuclear factors," Cardiovascular Research, vol. 72, no. 3, pp. 473-482, 2006.

[24] A. Keys, A. Mienotti, M. J. Karvonen et al., "The diet and 15year death rate in the seven countries study," American Journal of Epidemiology, vol. 124, no. 6, pp. 903-915, 1986.

[25] V. Konstantinidou, M.-I. Covas, D. Muñoz-Aguayo et al., "In vivo nutrigenomic effects of virgin olive oil polyphenols within the frame of the Mediterranean diet: a randomized controlled trial," The FASEB Journal, vol. 24, no. 7, pp. 2546-2557, 2010.

[26] M.-I. Covas, "Olive oil and the cardiovascular system," Pharmacological Research, vol. 55, no. 3, pp. 175-186, 2007.

[27] R. Estruch, M. A. Martínez-González, D. Corella et al., "Effects of a Mediterranean-style diet on cardiovascular risk factors: a randomized trial," Annals of Internal Medicine, vol. 145, no. 1, pp. 1-11, 2006.

[28] T. Weinbrenner, M. Fitó, R. De La Torre et al., "Olive oils high in phenolic compounds modulate oxidative/antioxidative status in men," Journal of Nutrition, vol. 134, no. 9, pp. 2314-2321, 2004.

[29] R. Solà, A. E. La Ville, J. L. Richard et al., "Oleic acid rich diet protects against the oxidative modification of high density lipoprotein," Free Radical Biology \& Medicine, vol. 22, no. 6, pp. 1037-1045, 1997.

[30] C. R. Sirtori, G. Franceschini, G. Gianfranceschi et al., "Activity profile of gemfibrozil on the major plasma lipoprotein parameters," European Journal of Epidemiology, vol. 8, supplement 1, pp. 120-124, 1992.

[31] F. Visioli, A. Poli, and C. Gall, "Antioxidant and other biological activities of phenols from olives and olive oil," Medicinal Research Reviews, vol. 22, no. 1, pp. 65-75, 2002.

[32] L. Calabresi, C. Banfi, C. R. Sirtori, and G. Franceschini, "Apolipoprotein A-II modulates HDL remodeling in plasma," Biochimica et Biophysica Acta-Lipids and Lipid Metabolism, vol. 1124, no. 2, pp. 195-198, 1992.

[33] J. S. Perona and V. Ruiz-Gutierrez, "Quantification of major lipid classes in human triacylglycerol-rich lipoproteins by highperformance liquid chromatography with evaporative lightscattering detection," Journal of Separation Science, vol. 27, no. 9, pp. 653-659, 2004.

[34] M. González-Santiago, J. Fonollá, and E. Lopez-Huertas, "Human absorption of a supplement containing purified hydroxytyrosol, a natural antioxidant from olive oil, and evidence for its transient association with low-density lipoproteins," Pharmacological Research, vol. 61, no. 4, pp. 364-370, 2010. 
[35] D. J. Gordon and B. M. Rifkind, "High-density lipoprotein-the clinical implications of recent studies," The New England Journal of Medicine, vol. 321, no. 19, pp. 1311-1316, 1989.

[36] J. A. Glomset, "The plasma lecithins: cholesterol acyltransferase reaction," The Journal of Lipid Research, vol. 9, no. 2, pp. 155-167, 1968.

[37] M. A. Kennedy, G. C. Barrera, K. Nakamura et al., "ABCG1 has a critical role in mediating cholesterol efflux to HDL and preventing cellular lipid accumulation," Cell Metabolism, vol. 1, no. 2, pp. 121-131, 2005.

[38] N. Wang, D. Lan, W. Chen, F. Matsuura, and A. R. Tall, "ATP-binding cassette transporters G1 and G4 mediate cellular cholesterol efflux to high-density lipoproteins," Proceedings of the National Academy of Sciences of the United States of America, vol. 101, no. 26, pp. 9774-9779, 2004.

[39] Y. Ji, B. Jian, N. Wang et al., "Scavenger receptor BI promotes high density lipoprotein-mediated cellular cholesterol efflux," The Journal of Biological Chemistry, vol. 272, no. 34, pp. 2098220985, 1997.

[40] H. Uto-Kondo, M. Ayaori, M. Ogura et al., "Coffee consumption enhances high-density lipoprotein-mediated cholesterol efflux in macrophages," Circulation Research, vol. 106, no. 4, pp. 779787, 2010 .

[41] M. Rosenblat, N. Volkova, R. Coleman, Y. Almagor, and M. Aviram, "Antiatherogenicity of extra virgin olive oil and its enrichment with green tea polyphenols in the atherosclerotic apolipoprotein-E-deficient mice: enhanced macrophage cholesterol efflux," Journal of Nutritional Biochemistry, vol. 19, no. 8, pp. 514-523, 2008.

[42] M. T. Montoya, A. Porres, S. Serrano et al., "Fatty acid saturation of the diet and plasma lipid concentrations, lipoprotein particle concentrations, and cholesterol efflux capacity," American Journal of Clinical Nutrition, vol. 75, no. 3, pp. 484-491, 2002.

[43] O. Helal, H. Berrougui, S. Loued, and A. Khalil, "Extra-virgin olive oil consumption improves the capacity of HDL to mediate cholesterol efflux and increases ABCA1 and ABCG1 expression in human macrophages," British Journal of Nutrition, vol. 109, no. 10, pp. 1844-1855, 2013.

[44] M. N. Franco, T. Galeano-Díaz, Ó. López et al., "Phenolic compounds and antioxidant capacity of virgin olive oil," Food Chemistry, vol. 163, pp. 289-298, 2014.

[45] S. Cicerale, L. J. Lucas, and R. S. J. Keast, "Antimicrobial, antioxidant and anti-inflammatory phenolic activities in extra virgin olive oil," Current Opinion in Biotechnology, vol. 23, no. 2, pp. 129-135, 2012.

[46] M. I. Covas, M. Fitó, R. M. Lamuela-Raventós, N. Sebastiá, C. De La Torre-Boronat, and J. Marrugat, "Virgin olive oil phenolic compounds: binding to human low density lipoprotein (LDL) and effect on LDL oxidation," International Journal of Clinical Pharmacology Research, vol. 20, no. 3-4, pp. 49-54, 2000.

[47] R. M. Lamucla-Raventós, M.-I. Covas, M. Fitó, J. Marrugat, and M. C. de la Torre-Boronat, "Detection of dietary antioxidant phenolic compounds in human LDL," Clinical Chemistry, vol. 45, no. 10, pp. 1870-1872, 1999.

[48] Á. Hernáez, S. Fernández-Castillejo, M. Farràs et al., "Olive oil polyphenols enhance high-density lipoprotein function in humans: a randomized controlled trial," Arteriosclerosis, Thrombosis, and Vascular Biology, vol. 34, no. 9, pp. 2115-2119, 2014.

[49] F. Pazzucconi, L. Mannucci, L. Mussoni et al., "Bezafibrate lowers plasma lipids, fibrinogen and platelet aggregability in hypertriglyceridaemia," European Journal of Clinical Pharmacology, vol. 43, no. 3, pp. 219-223, 1992.
[50] H. Y. Chung, M. Cesari, S. Anton et al., "Molecular inflammation: underpinnings of aging and age-related diseases," Ageing Research Reviews, vol. 8, no. 1, pp. 18-30, 2009.

[51] K. Trudel, D. Sinnett, R. W. James et al., "Iron-ascorbic acidinduced oxidant stress and its quenching by paraoxonase 1 in HDL and the liver: comparison between humans and rats," Journal of Cellular Biochemistry, vol. 96, no. 2, pp. 404-411, 2005.

[52] M. Sevov, L. Elfineh, and L. B. Cavelier, "Resveratrol regulates the expression of LXR-alpha in human macrophages," Biochemical and Biophysical Research Communications, vol. 348, no. 3, pp. 1047-1054, 2006.

[53] H. Berrougui, G. Grenier, S. Loued, G. Drouin, and A. Khalil, "A new insight into resveratrol as an atheroprotective compound: inhibition of lipid peroxidation and enhancement of cholesterol efflux," Atherosclerosis, vol. 207, no. 2, pp. 420-427, 2009. 


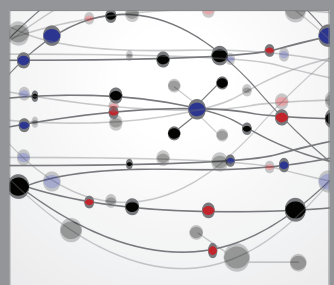

The Scientific World Journal
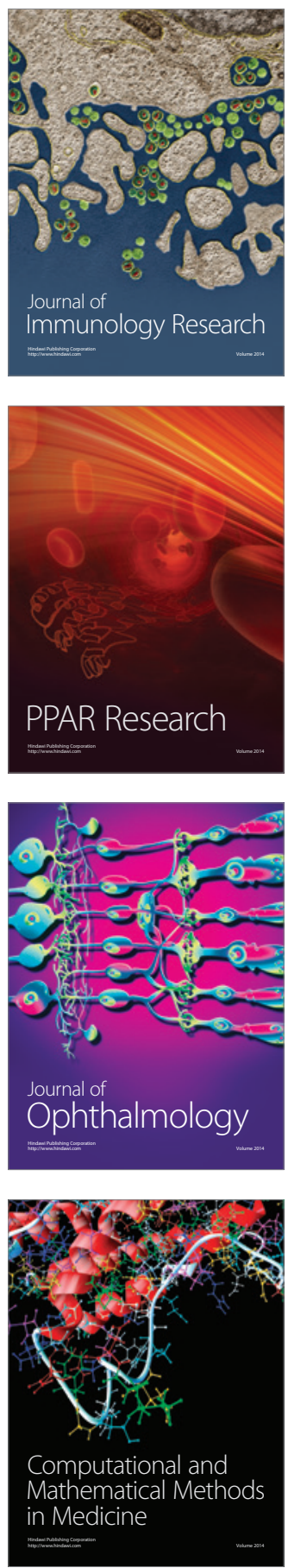

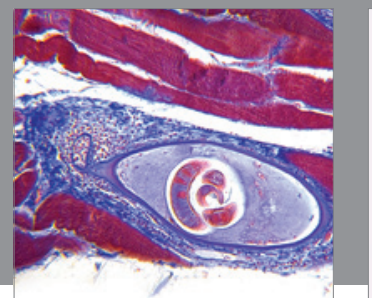

Gastroenterology

Research and Practice
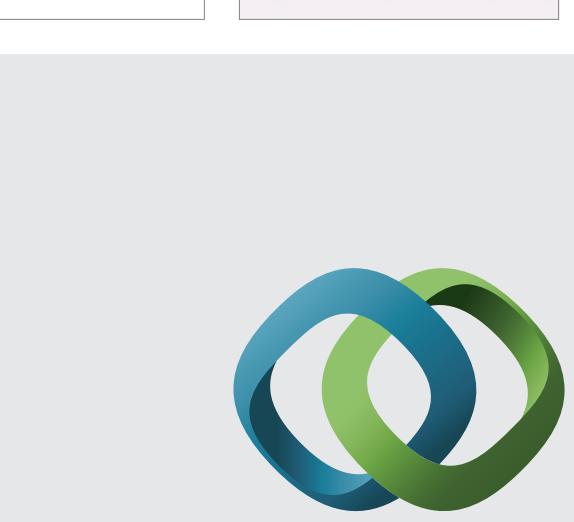

\section{Hindawi}

Submit your manuscripts at

http://www.hindawi.com
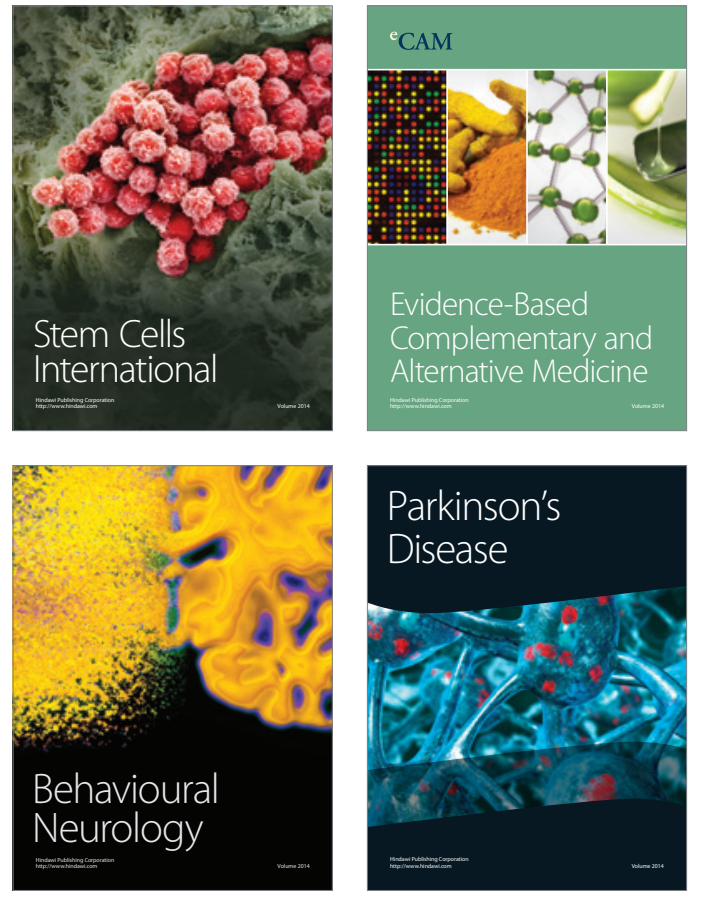
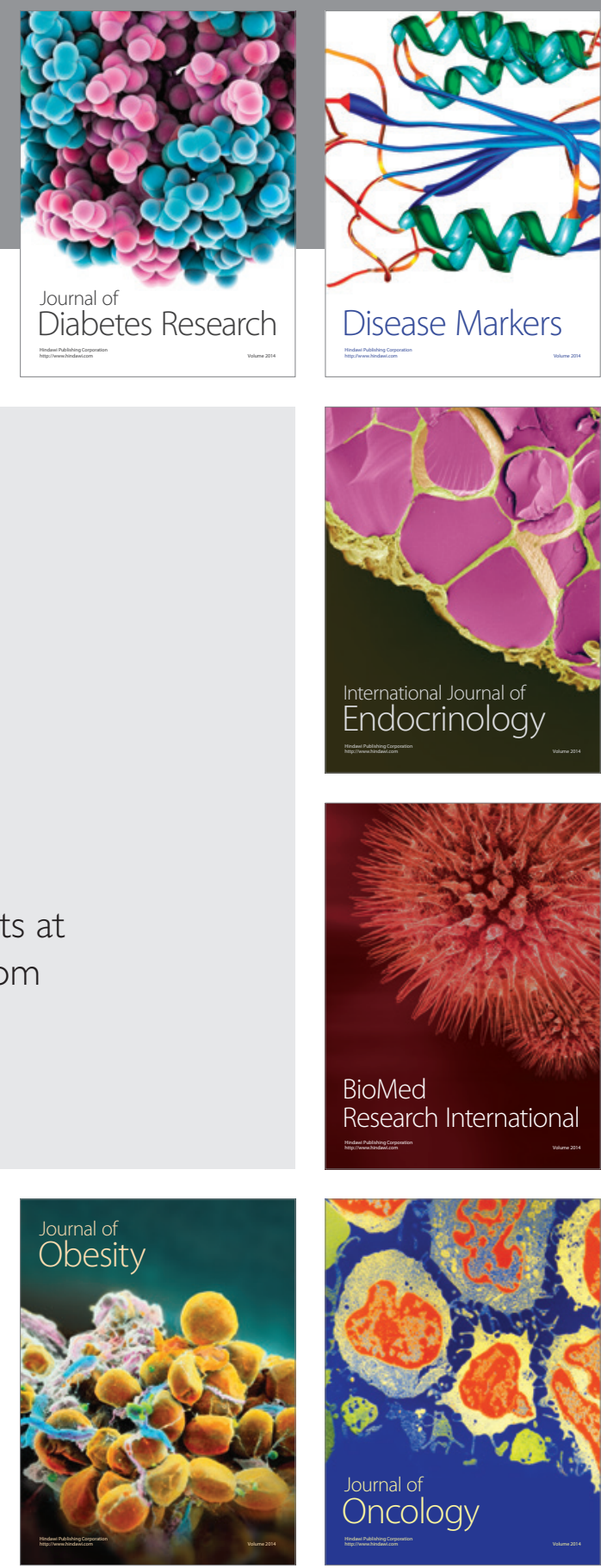

Disease Markers
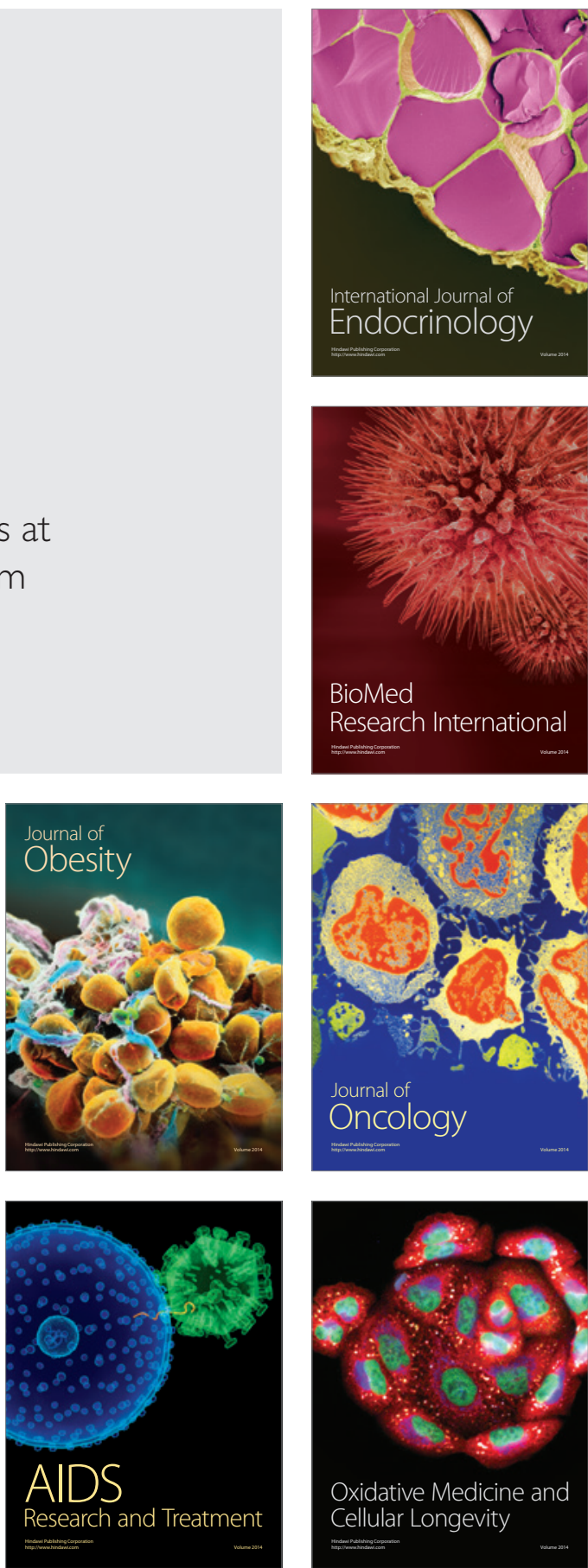